\title{
Simulation Fluidity Test for Semisolid Squeeze Casting
}

\author{
Richard Pastirčák ${ }^{1, *}$, and Denis Martinec ${ }^{1}$ \\ ${ }^{1}$ Department of technological engineering, University of Žilina, Univerzitná 8215/1, 01026 Žilina
}

\begin{abstract}
The paper deals with semi solid squeeze casting technology. Fluidity tests were designed for the selected technology. The shape of the test casting was designed in the shape of test bars with different thicknesses and also in the shape of a stepped casting. The thickness of the individual elements was chosen on the basis of a selected / preferred numbers R10 EN STN 17. As a result, the thickness of the elements was 2.0, 2.5, 3.15, 4.0, 5.0 and $6.3 \mathrm{~mm}$. Designed fluidity tests were verified by using ProCast simulation software. The selected process parameters were: operating pressure $80 \mathrm{MPa}$, mold temperature $200^{\circ} \mathrm{C}$, piston speed $30 \mathrm{~mm} \cdot \mathrm{s}^{-1}$. The experimental material was an AlSi7Mg0.3 alloy with a different solid phase content. The initial solid amount were 50, 55 and $60 \%$. The effect of solidus and liquidus, temperature distribution and pressure was monitored during the evaluation of fluidity.
\end{abstract}

\section{Introduction}

Semi-solid metal processing technologies use the thixotropic behavior of metallic materials. The thixotropic behavior is manifested only in the initial material with a non-dendritic structure. Over the years, various methodologies have been used to achieve non-dendritic microstructure. Although the potential of this process is obvious and its long history has seen numerous attempts at full commercialization, only a limited number have been successfully exploited. Methodologies for achieving the necessary non-dendritic structures are key to the successful commercialization of these processes [1, 2, 3].

In contrast to the mentioned processes, with the semi solid squeeze casting (SSSC) technology, the standard alloy is heated to a temperature between liquid and solid. Initial material in the semi-solid state is thus characterized by a dendritic structure. The dendritic structure causes resistance to flow when filling the mold cavity. The resistance affect not only the flow itself, but also the pressure distribution in the individual sections of the casting, which results in different mechanical properties in the cross sections of the casting. For these reasons, it is important for the research of SSSC technology to know the foundry properties of the material, especially the fluidity. Fluidity is one of the most important foundry properties, which determines the limits of the technology used $[4,5,6]$.

The vast majority of fluidity tests involve a controlled metal flow of known composition and temperature into a mold cavity of known temperature and dimensions.

\footnotetext{
${ }^{*}$ Corresponding author: richard.pastircak@,fstroj.uniza.sk
} 
After solidification, the length of the resulting sample is measured and evaluated as the fluidity of given metal.

Most of fluidity tests are performed in one of three ways: The metal is poured into a spiral mold or into a cavity (cavities) with long thin sections, extracted from a heated crucible by vacuum or extruded from a pressure die casting machine into a "zigzag" mold. In each case, the degree of fluidity is the length of the casted sample, with respect to a specific parameters (melt temperature, mold material, mold coating, mold temperature, or other experimental conditions). These parameters must be precisely determined and controlled in order to obtain equivalent results $[1,7]$.

In all three cases, a microstructural cross-sectional evaluation is also used, especially near the end of the casting, to examine how the solidification mechanisms "dampened" the flow.

Commonly, fluidity tests are designed for gravity casting technologies, but these tests are not defined for increased forces acting during mold filling. For this reason, we have proposed a fluidity test, which will evaluate, in addition to the length of the filled element, also the increase in mechanical properties, especially the ductility of the casting material $[4,8]$.

\section{Experiment methodology}

To verify the results of the numerical simulation and the experimental casting, both results were evaluated and compared. For a correct calculation, it is necessary to know the actual curve of the solid phase fraction depending on the temperature. The temperature dependence of the solids fraction was obtained by thermal analysis. Figure 1 shows the experimentally obtained results and the solid phase fraction amount inserted into the simulation program Figure 2.

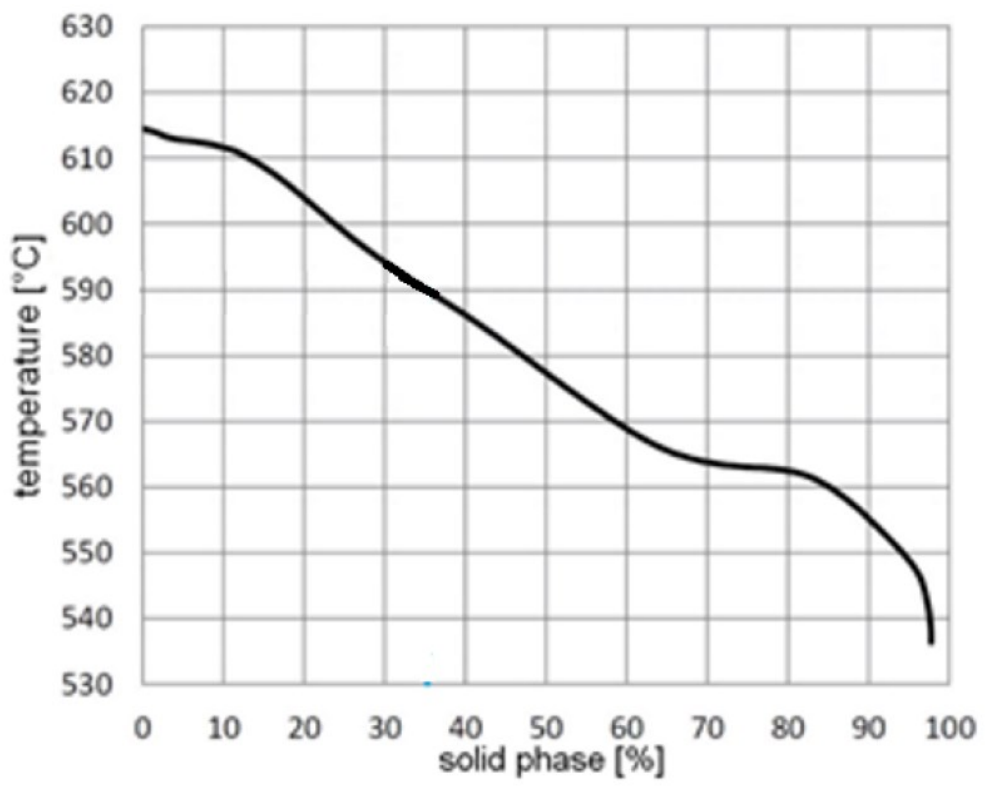

Fig. 1. Experimentally measured solid fraction. 


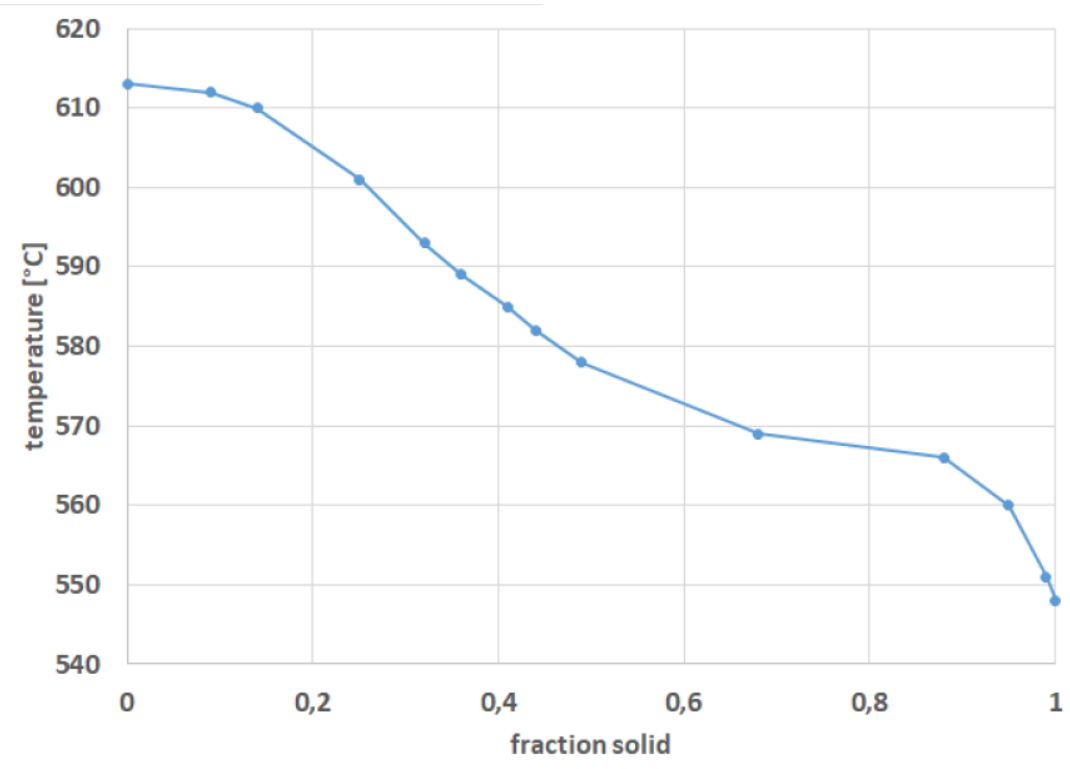

Fig. 2. Solid fraction for simulation calculation.

Aluminum alloy AlSi7Mg0.3 was used as experimental material. The chemical composition of used alloy is described in Table 1. Alloy was chosen due to its wide solidification interval, which provides relatively easy way to obtain different ratios of solid and liquid phases by changing the temperature.

Table 1. Chemical composition of the AlSi7Mg0.3 alloy.

\begin{tabular}{|c|c|c|c|c|c|c|}
\hline $\mathbf{S i}$ & $\mathbf{M g}$ & $\mathbf{M n}$ & $\mathbf{C u}$ & $\mathbf{F e}$ & $\mathbf{Z n}$ & $\mathbf{T i}$ \\
\hline 8.835 & 0.303 & 0.006 & 0.007 & 0.135 & 0.004 & 0.002 \\
\hline
\end{tabular}

Based on the performed experiment, a numerical simulation calculation was performed in the ProCast software. The basic parameters of the casting process are given in Table 2.

Table 2. Process parameters for experimental casting.

\begin{tabular}{|c|c|c|c|c|}
\hline $\begin{array}{c}\text { Temperature } \\
\text { alloy } \\
{\left[{ }^{\circ} \mathbf{C}\right]}\end{array}$ & $\begin{array}{c}\text { Fraction solid } \\
{[\%]}\end{array}$ & $\begin{array}{c}\text { Temperature die } \\
{\left[{ }^{\circ} \mathbf{C}\right]}\end{array}$ & $\begin{array}{c}\text { Applied } \\
\text { pressure } \\
{[\mathbf{M P a}]}\end{array}$ & $\begin{array}{c}\text { Piston movement } \\
\text { speed } \\
{\left[\mathbf{m m}^{-1} \mathbf{s}^{-1}\right]}\end{array}$ \\
\hline $580 \pm 2$ & 50 & $200 \pm 5$ & 30 & 30 \\
\hline
\end{tabular}

During the calculation, the boundary conditions of the simulation were varied until a satisfactory agreement with the experimental casting was achieved. Subsequently, these boundary conditions were used in the simulation calculations of the proposed castings for the semi-solid fluidity test.

During the design of the combined fluidity test, the shape of the test casting was chosen with regard to its purpose - evaluation of mechanical properties. The aim of the proposed shape was a casting that would give two pieces of information. The first is to obtain information about the filling of the mold cavity with different geometries. Secondly, information on the influence of pressure on the microstructure was also important. For this reason, castings with different element thicknesses have been designed. Stepped casting and casting with separate arms thickness. The shape of the stepped casting is shown in Figure 3 
and the shape of a casting with separate arms in Figure 4. the thickness of the elements was the same in both cases, which was chosen on the basis of selected numbers R10 EN STN 17 with the sequence $2.0,2.5,3.15,4.0,5.0,6.3 \mathrm{~mm}$.

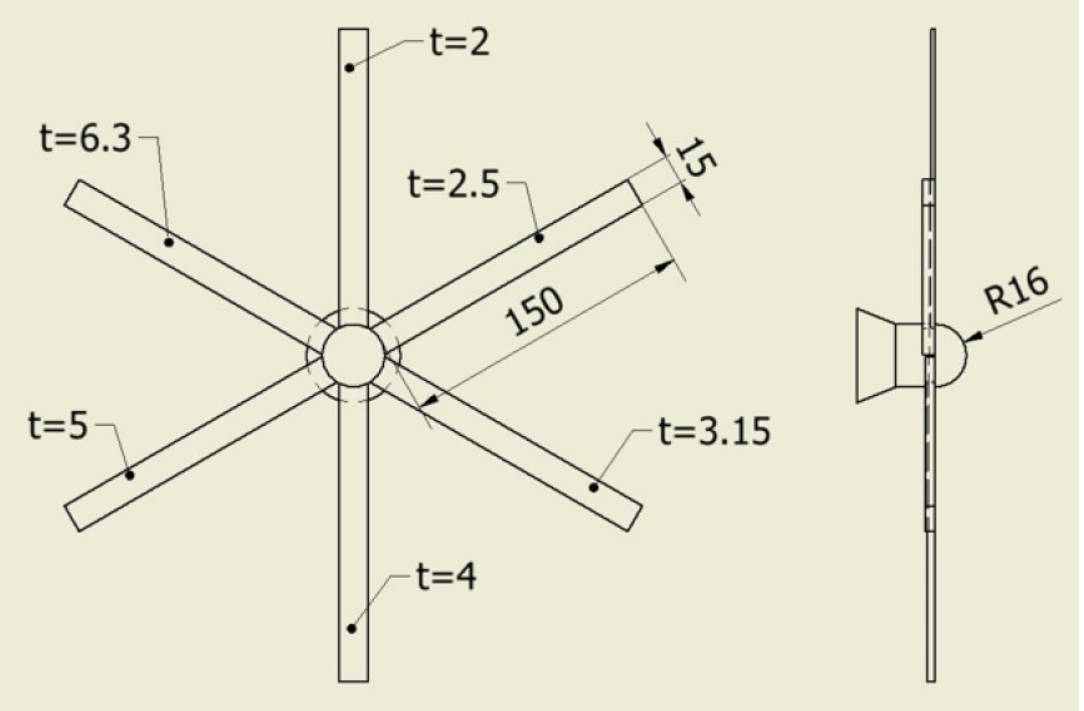

Fig. 3. Casting with separate arms.

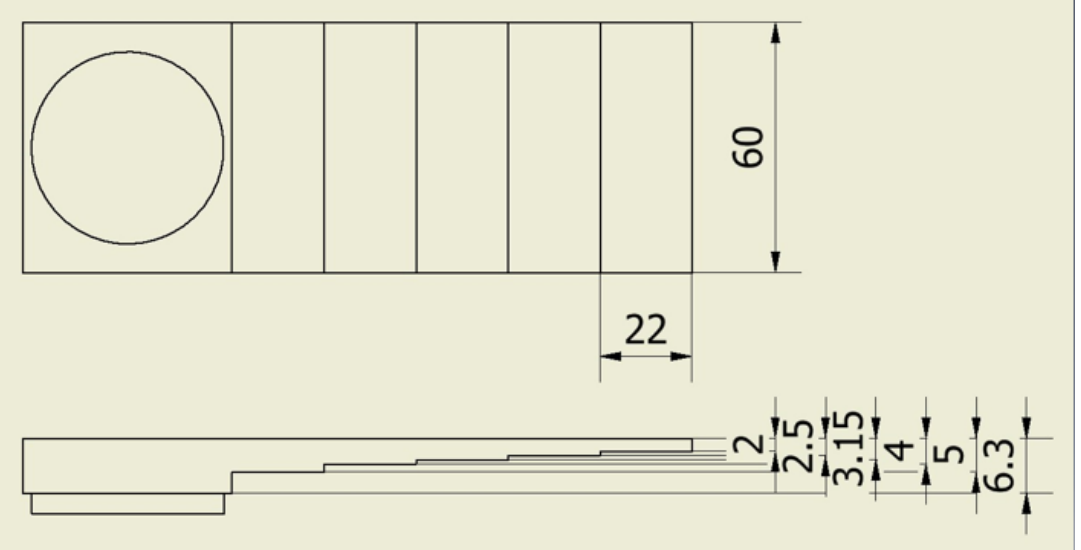

Fig. 4. Stepped casting.

\section{Results and discussion}

A comparison of the results of the numerical simulation and the experimental casting is shown in Figure 5. 
Temperature $[\mathrm{I}$

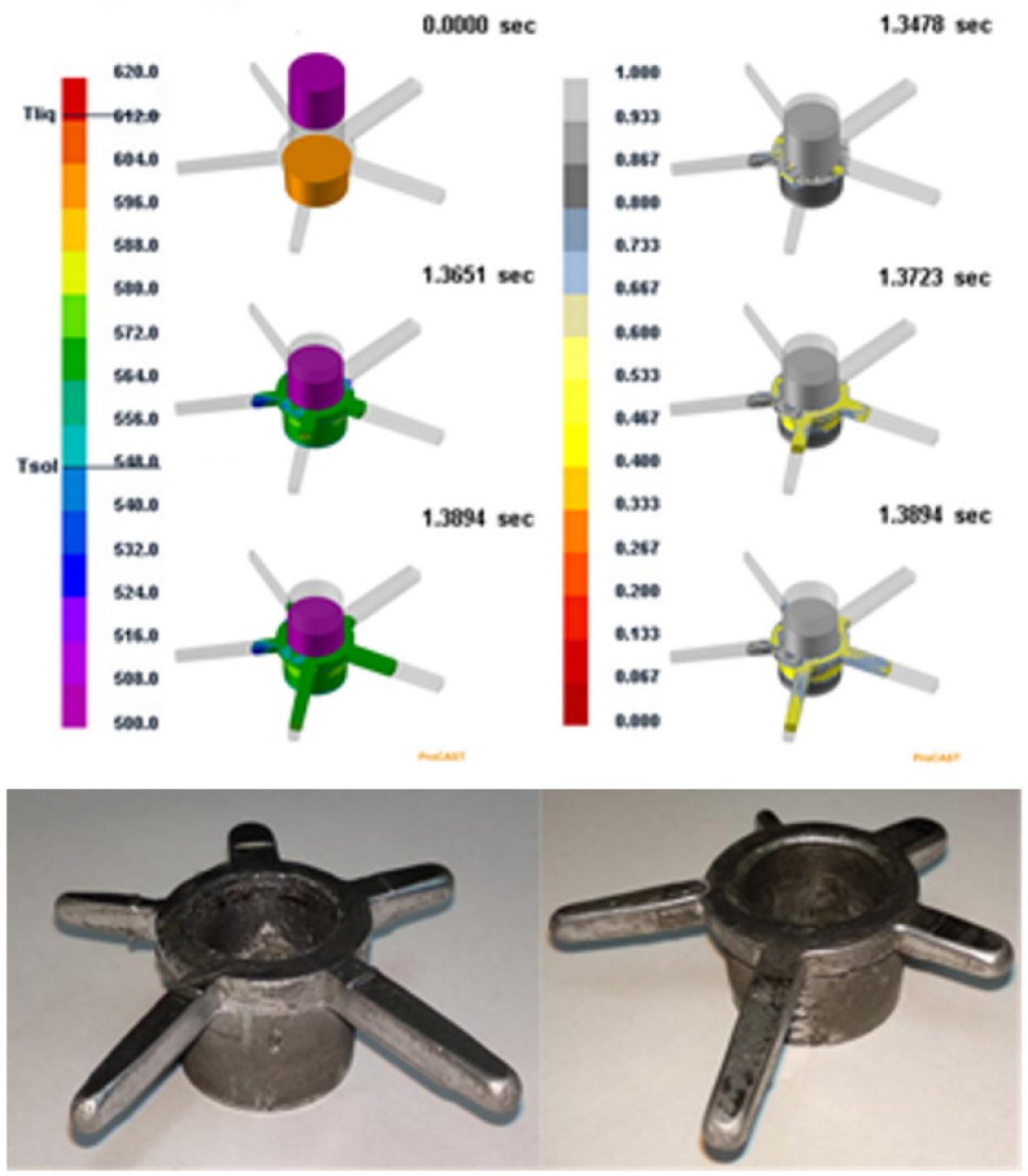

Fig. 5. Results of the numerical simulation and the experimental casting.

By changing the process parameters, it is possible to achieve better filling of the element, but the pressure distribution in the semi-solid state is complicated, what can be observed on experimental casting. The thin part with a thickness only $2 \mathrm{~mm}$ was not filled due to the high cooling capacity of the mold. This phenomenon is also manifested by the gradual elongation of thicker elements. Since a low operating pressure of $30 \mathrm{MPa}$ was chosen during the experiment, a shortening of the $5 \mathrm{~mm}$ and $6 \mathrm{~mm}$ length elements can be observed. This is due to the reduction of the specific pressure in the cross section, and thus the effect of the applied pressure is not manifested.

In Figure 6 shows the results of a simulation calculation for a test bars with different thicknesses with a $60 \%$ solid fraction. The results for a solid fraction of $55 \%$ are shown in Figure 7, for a solid fraction of $50 \%$ are shown in Figure 8. 


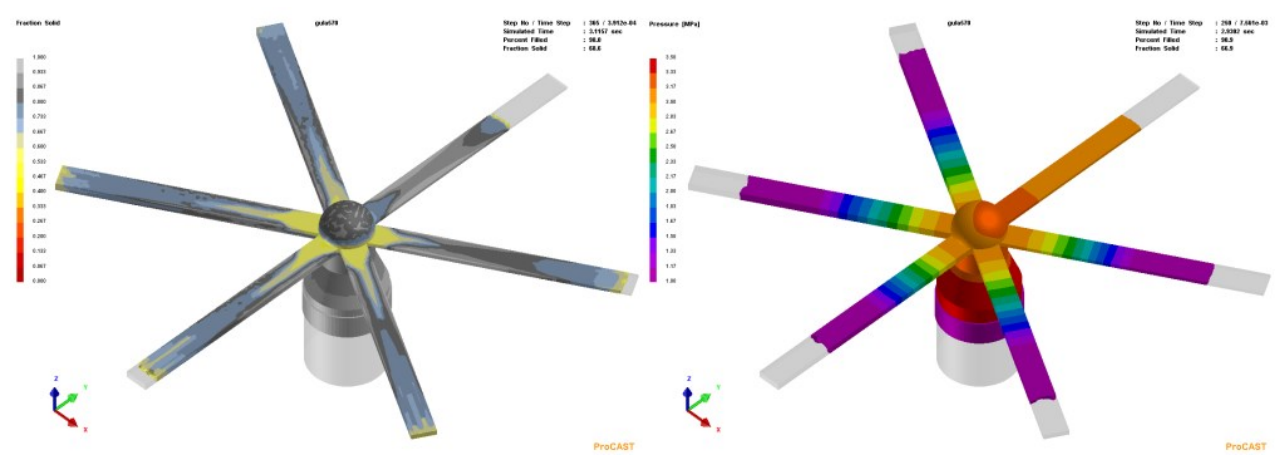

Fig. 6. Test bars with different thicknesses with a $60 \%$ solid fraction.

With a solidus amount of $60 \%$ during the pouring, elements with a thickness of 3.15 , 5.0 and $6.3 \mathrm{~mm}$ are filled along their entire length. With an element thickness of $2.0 \mathrm{~mm}$, the element was filled to the extent of two thirds of the total length. The flow was stopped due to cooling below the solidus temperature at the beginning of the element. Elements with a thickness of 2.5 and $4.0 \mathrm{~mm}$ were not completely filled due to the solidification at the central parts of these elements. The total filling of the casting is $98 \%$. The pressure distribution at the moment of flow freeze in the thinnest element indicates a slight effect of the cross-section on the mentioned pressure distribution.

solid fraction of $50 \%$ are shown in Figure 8.

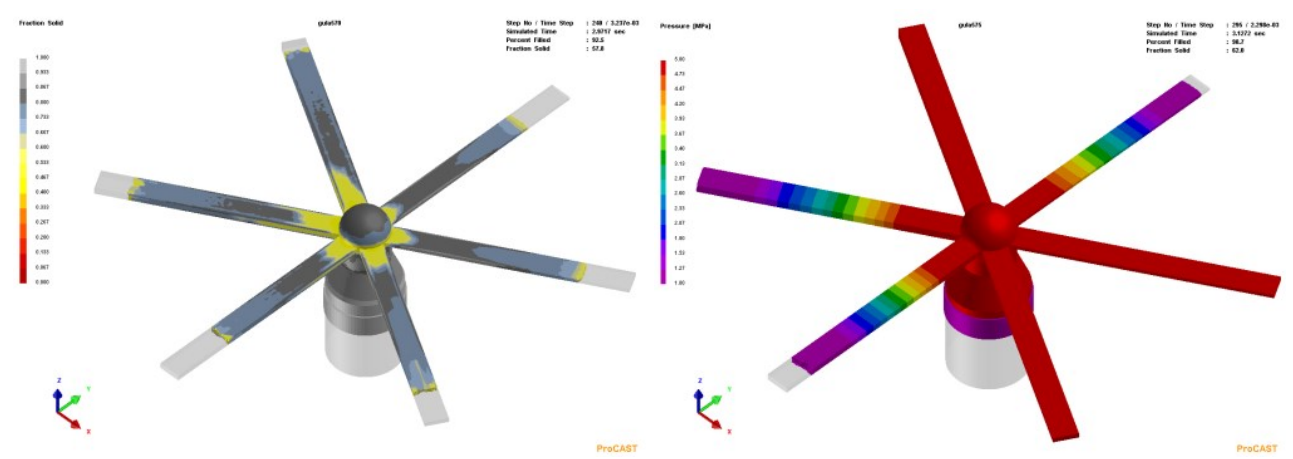

Fig. 7. Test bars with different thicknesses with a $55 \%$ solid fraction.

The temperature distribution and the proportion of solid phase during the filling of the casting correspond to the thickness of the elements and the amount of accumulated heat. The total filling of the casting is $99.7 \%$. Failure did not occur only in the element with a thickness of $4.0 \mathrm{~mm}$. It follows from the pressure distribution that this phenomenon was influenced by the position of the element relative to the thinnest part of the casting. 


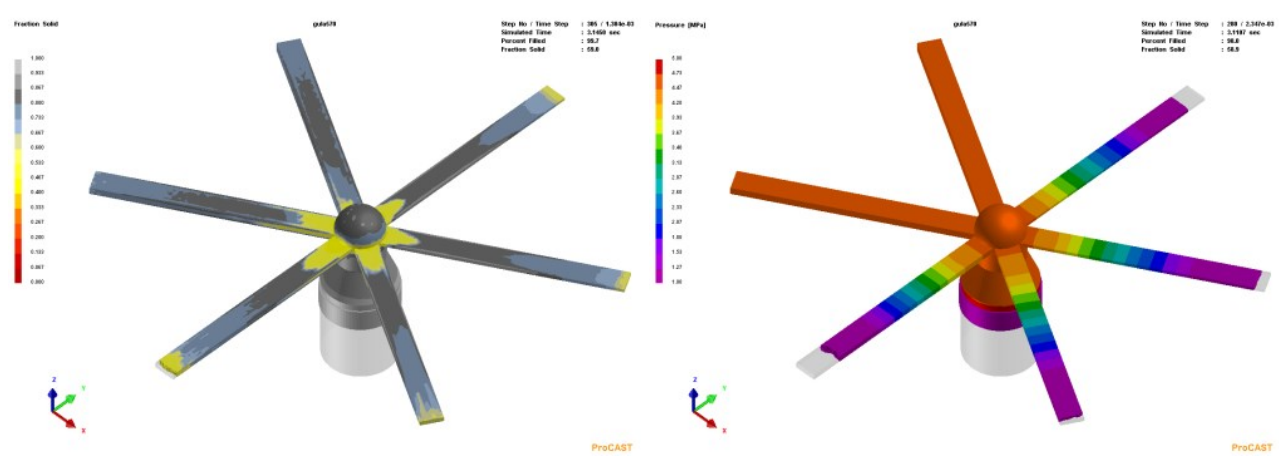

Fig. 8. Test bars with different thicknesses with a $50 \%$ solid fraction.

At $50 \%$ amount of solid phase, the process is similar to $55 \%$ solid phase amount. The only difference is that the higher initial temperature reduces the effect of the pressure distribution and the filling of the mold cavity corresponds to the thickness of the individual elements a stepped casting.

The results of the simulation calculation for a stepped casting are shown in Figure 9 and Figure 10.

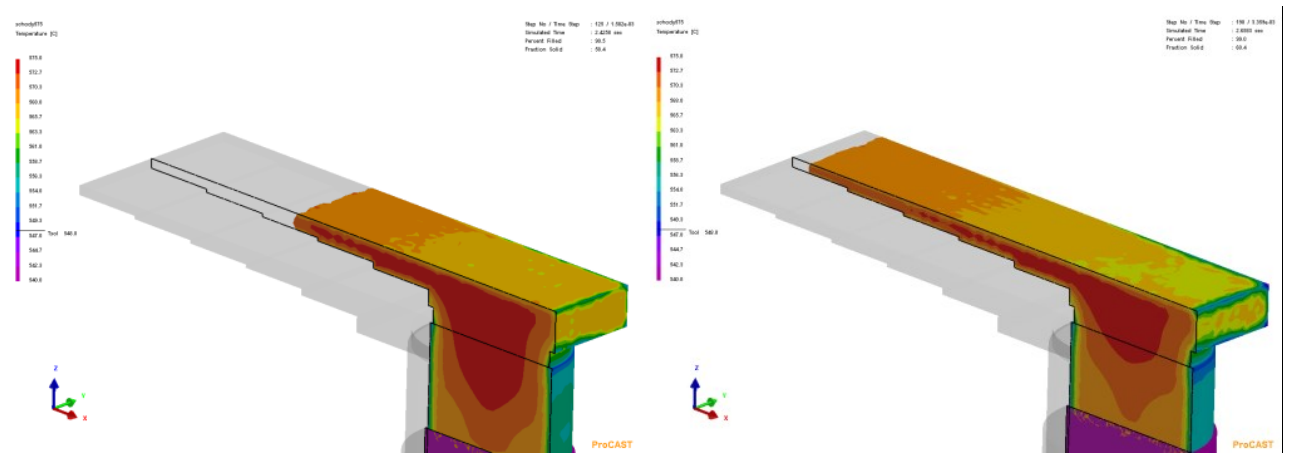

Fig. 9. Stepped casting with a $60 \%$ solid fraction.

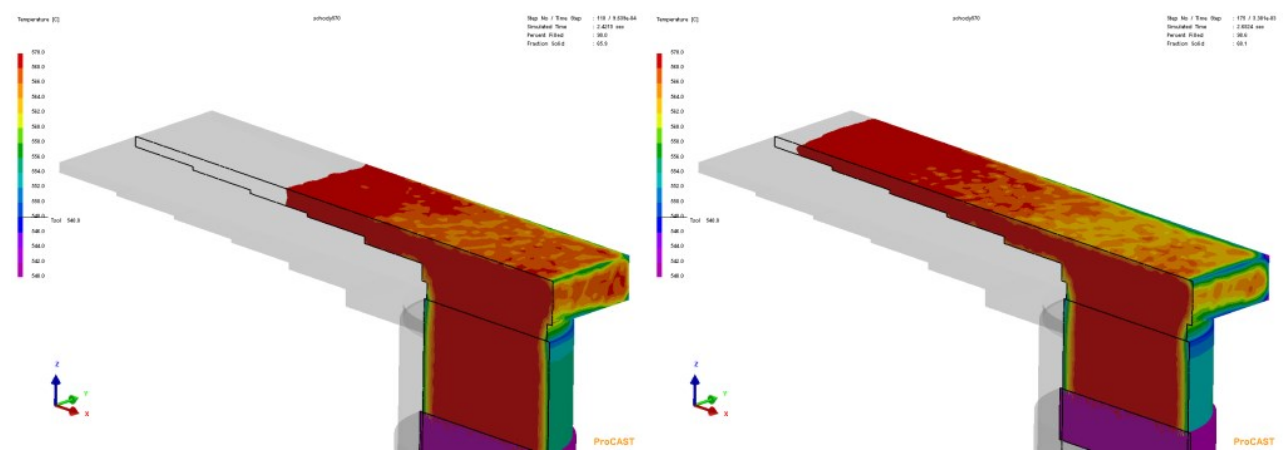

Fig. 10. Stepped casting with a $55 \%$ solid fraction.

The results of the numerical simulation calculation of the stepped casting were not interesting at the $50 \%$ amount of the solid phase. Filling of the individual elements was smooth and no unpredictable phenomena were observed. With a solids amount of $50 \%$ at the beginning of the filling, it can be observed that the cooling rate of the mold is high. The temperature of the melt flow decrease below $30 \%$ of the liquid phase, which is the liquidity yield of the used material. A higher temperature at $55 \%$ initial amount of solid 
results in less effect on casting performance. Nevertheless, the thinnest part with a thickness of $2.0 \mathrm{~mm}$ was not filled. In both cases, the total filling of the casting was $99 \%$. no irregularities were observed during the pressure in all cases.

\section{Conclusion}

When choosing a fluidity test for technologies with a higher operating pressure during the filling of the mold cavity, it is advisable to choose different elements. For most technologies with a higher applied pressure, a test casting with separated elements has a higher informative value. The separated elements of the casting give more information about the filling conditions. For example, they allow to set technology limits under selected technological conditions. A similar results of the given issue can be seen in Ramadan [9].

Squeeze casting or semi solid squeeze casting technology is suitable for thicker castings. The possibility of applying this technology for complex castings is characterized by a stepped element. The casting shape makes it possible to observe the interaction between the different thicknesses of the casting. The simulation model in the ProCast program is in accordance with the results of the authors Fu and Wang [10] in the field of metal flow simulation in the semi-solid state.

Based on the results, it will be necessary to select an odd number of elements when designing a fluidity test in order to eliminate undesirable phenomena caused by the mutual influence of opposing arms.

Acknowledgements: This work was created in the framework of the grant project VEGA $\mathrm{N}^{\circ}$ $1 / 0494 / 17$. The authors acknowledge the grant agency for support.

\section{References}

1. G. Li, H. Lu, X. Hu, F. Lin, X. Li, Q. Zhu, Metals 2020, 10, 238; doi:10.3390/met10020238

2. R. Lenhard, K. Kaduchová, Š. Papučík, J. Jandačka. Utilization of heat pipes for transfer heat from the flue gas into the heat transfer medium. EPJ web of conferences. 2014, 67, 02067. ISSN 2101-6275

3. R. Podprocká, D. Bolibruchová, Archives of Foundry Engineering, 17 (3), 217-221 (2017)

4. R. Pastircak, J. Scury, M. Bruna, D. Bolibruchova, Archives of Foundry Engineering, 17 (2), 75-78 (2017)

5. L. Richtarech, D. Bolibruchova, M. Bruna, Archives of Foundry Engineering, 15 (2), 95-98, (2015)

6. J. Winczek, M. Gucwa, M. Mičian, R. Koňár, S. Parzyh, Arch. Metall. Mater., 64 (3), 1111-1115 (2019)

7. L. Jakubovicova, B. Ftorek, V. Baniari, et al., MMS 2016, Procedia Engineering, 177, 520-525 (2016)

8. K. Major-Gabrys, A. Grabarczyk, St. M. Dobosz, Metalurgia, 55 (3), 385-387 (2016)

9. M. Ramadan, N. El-Bagoury, J Mater Sci (2011) 46:4013-4019 DOI 10.1007/s10853011-5329-7

10. J. Fu, K. Wang. Procedia Engineering, 81, 1565-1570 (2014) 